\title{
Computational prediction of out-of-plane welding distortion and experimental investigation
}

\author{
D Camilleri, T Comlekci, and T G F Gray* \\ Department of Mechanical Engineering, University of Strathclyde, Glasgow, Scotland, UK \\ The MS was received on 19 February 2004 and was accepted after revision for publication on 30 September 2004.
}

DOI: $10.1243 / 030932405 X 7809$

\begin{abstract}
The main aim of the work was to investigate a simplified finite element simulation of the out-of-plane distortion caused by fusion butt welding. The thermal transient part of the simulation made use of a finite element analysis of the two-dimensional cross-section of the weld joint and the thermoelastic-plastic treatment was based on analytical algorithms describing transverse and longitudinal deformations, leading to predictions of transverse angular deformation and longitudinal contraction force. These results were then applied to a non-linear elastic finite element model to provide predictions of the final angular and overall deformations of the butt-welded plates. The validity of the simulation was investigated via full-scale tests on $4 \mathrm{~m} \times 1.4 \mathrm{~m} \times 5 \mathrm{~mm}$ steel plates, butt welded using a flux-cored $\mathrm{Ar}-\mathrm{CO}_{2}$ metal-inert gas process. Thermography and thermocouple arrays were used to validate the thermal transient computations and out-of-plane deformations were measured using displacement transducers for transient deformations and a laser scanning system to measure the profiles of the whole plates before and after welding. The results of six full-scale tests are given and comparison with the simulations shows that the procedure provides good prediction of the angular and overall out-of-plane deformations. Prediction accuracy requires account to be taken of initial shape, gravity loading, and support conditions.
\end{abstract}

Keywords: welding distortion, welding simulation, butt welds, out-of-plane welding deformation, angular deformation

\section{INTRODUCTION}

\subsection{Background}

Many industries concerned with the fabrication of thin-plate structures experience difficulties and high rectification costs related to cutting and welding thermal distortion. A recent review of a large collaborative programme, funded by the US Navy Office of Naval Research [1] states: 'Severe distortions have emerged as a major obstacle to the cost-effective fabrication of such lightweight structures.' Out-ofplane distortion, in particular, makes it difficult to align the edges of subassemblies and results in a lack of 'fairness' in the completed structure. The general practice in such industries is to base predictions of distortion on previous outcomes of particular

\footnotetext{
${ }^{*}$ Corresponding author: Department of Mechanical Engineering, University of Strathclyde, James Weir Building, 75 Montrose Street, Glasgow G1 1XJ, UK; email: tom.gray@strath.ac.uk
}

cutting, welding, and assembly sequences, but this is not always successful. A more systematic version of this approach has been reported recently [2], based on neural network software. However, there are few predictive tools at present to support design and manufacturing optimization of such structures with respect to structural function and manufacturing variables. Various analytical and computational approaches to the problem are possible and have become more applicable as computational capability increases, but such strategies have not so far been widely used in industries concerned with fabrication of this type, due presumably to complexity and the need for in-house expertise.

The overall project, of which this study is a part, aims to improve the applicability of computational distortion prediction by providing simple and adaptable methodologies, which can be readily validated through experience of application in an industrial context. A key factor that underlies this strategy is an uncoupled computational procedure, whereby 
the thermal transient, thermoelastic-plastic and structural stages of the thermomechanical deformations induced by fusion welding are treated separately. The theoretical models and results generated in the project have been supported at all stages by welding tests of a realistic nature to maintain confidence in the validity and applicability of the treatments. This approach has been demonstrated in reference [3] where experimental and computational results were given for the welding distortion of a pilot test series of small butt-welded steel plates $(0.5 \mathrm{~m} \times 0.5 \mathrm{~m}$ and $6 \mathrm{~mm}$ thick). The present paper deals with aspects of a subsequent test series, where large steel plates (nominally $4 \mathrm{~m} \times 1.4 \mathrm{~m}$ and $5 \mathrm{~mm}$ thick) were butt welded using similar procedures. This scale of test piece is realistic in relation to common industrial practice and explores further complexities not present in the smaller models, such as buckling phenomena, the effects of gravity and support positions, and variations in pre-welding assembly practices.

\subsection{Outline of the uncoupled computational approach}

The first step in any computation of welding distortion is to establish the transient thermal distributions caused by a given welding process in the geometry of interest. This may be achieved in a variety of ways, ranging from the early analytical treatments of Rosenthal [4] to finite-element-based methods, as reviewed recently by Lindgren [5]. In the present approach, it is argued that the main characteristics of the thermal transients that drive typical distortion processes can be captured through relatively simple treatments. In particular, it does not appear to be necessary to model the precise details of the thermal patterns close to the molten weld pool. This is fortunate, as the temperature-dependent thermal properties of the materials involved are usually inaccurately known and the thermal outcomes of practical welding processes are both difficult to predict and subject to variation in application. Reference [6] from the earlier pilot study of small plates showed that a simple thermal transient treatment based on a two-dimensional cross-section of the weld was sufficient to provide accurate temperature distributions, which correlated with experimental data based on thermocouple and thermographic measurements. This approach was carried over to the present tests on large plates, where the transients were also checked experimentally, as shown in Fig. 1 (the non-dimensionalization shown for the transverse positions of the thermocouples will be discussed later).

Although a purely computational approach can be used to provide the required thermal information, it is likely that some measure of application-specific measurement will usually be required to index the thermal transient calculations to the actual transient temperature fields during welding operations. The main driving parameters relevant to thermal transients are the heat input rate and the material thermal properties, but variations in the process environment mean that a 'weld efficiency' factor is usually necessary to

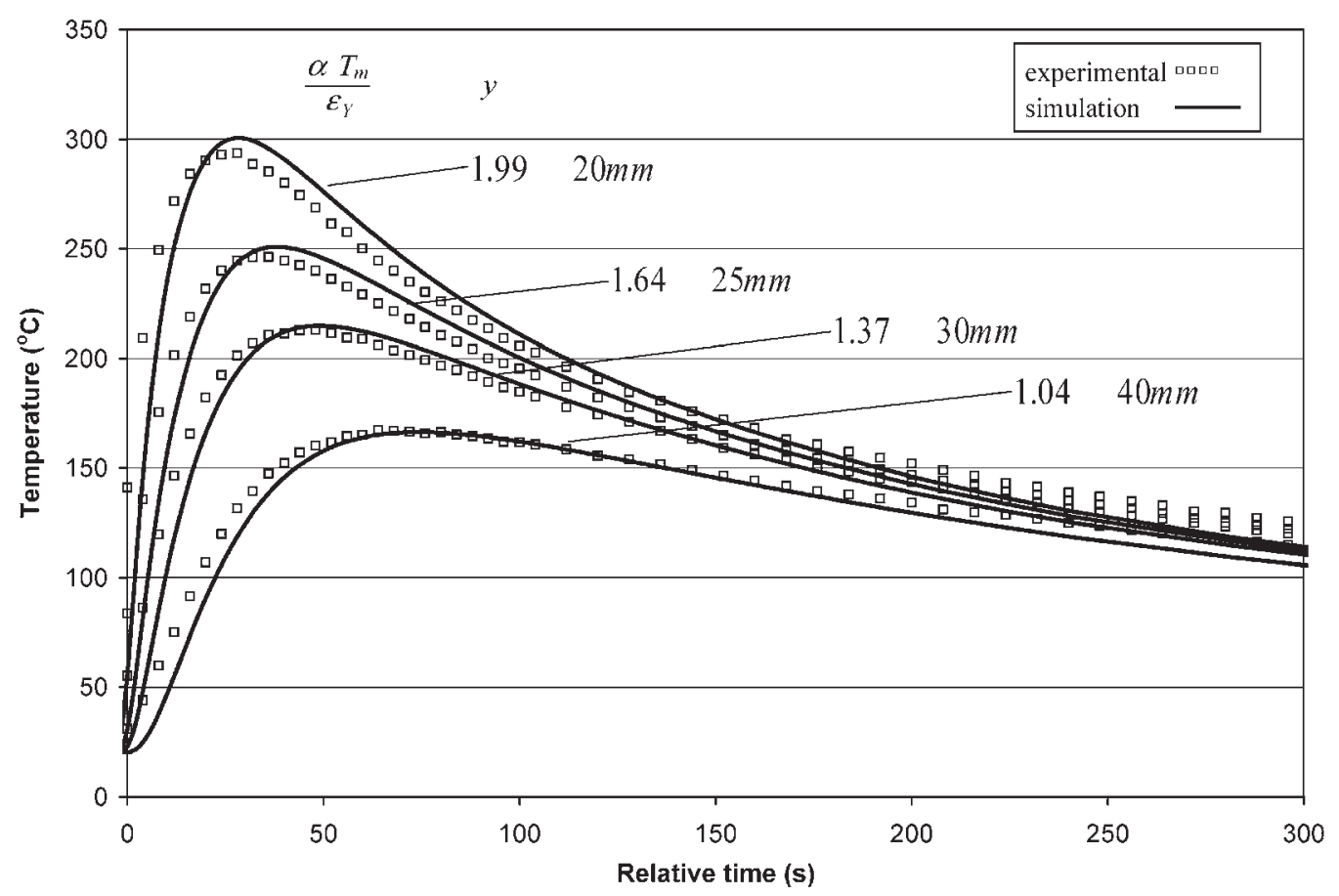

Fig. 1 Comparison of thermocouple results and two-dimensional transient model 
relate the calculation to reality. Thermographic imaging, as demonstrated in reference [6], provides a practical approach to determining such correlations and this tool has now developed to the point where it is readily applicable to industrial cases.

In the second computational stage of the uncoupled approach, differential strains induced by the transient thermal gradients are translated into contraction stresses, which produce mechanical distortion. This is the most intractable and complex part of the whole procedure, not least because the required high-temperature material properties are usually poorly known and the material also changes state. The algorithms used in the present treatment to solve this stage of the process have benefited from the early insights of Okerblom [7]. He noted various typical features of the thermomechanical transients generated by fast-moving fusion welding heat sources and made several bold simplifications on the strength of these observations. The availability of new computational methods means that some of these simplifications are no longer necessary, but others remain very powerful as ways to avoid the problematic aspects described above.

The main aim of this paper, therefore, is to delineate the terms of a simplified approach to the thermoelastic-plastic stage within the welding distortion process and to examine critically the main assumptions in terms of the mechanical behaviour of fullsize butt-welded steel plates. Although the validation is specific to this problem and material, the conclusion should carry over to other situations that can be characterized by similar thermal transient patterns.

\section{SIMPLIFIED ANALYTICAL MODELS OF THE THERMOMECHANICAL PROCESS}

\subsection{Longitudinal welding deformations}

Okerblom [7] recognized that the major irreversible deformations responsible for distortion are driven by the cooling phase of the process in the region behind the travelling heat source. The thermal gradients transverse to this line are typically steep, whereas the gradients parallel to the weld are relatively gradual, as can be deduced from Fig. 1. This fact suggested a simple treatment for longitudinal contraction, in terms of a transverse plane strain slice, which is passed through the quasi-stationary temperature field.

Further simplification is made by assuming that the elastic longitudinal stress developed during cooling at a given transverse location will depend in the first instance on the maximum thermal strain $\alpha T_{\mathrm{m}}$ generated at that transverse coordinate, irrespective of the relative longitudinal coordinate where that occurs
( $T_{\mathrm{m}}$ is the maximum temperature relative to the reference temperature, taken as zero). The analytical treatment arising from this simplification will be designated the 'mismatched thermal strain' (MTS) algorithm. It can be seen from Fig. 1 that regions nearer the centre of the slice begin to start to cool before adjacent regions further away from the centre and they cool more rapidly, eventually converging to a common rate. The resulting thermal mismatches develop contraction forces, progressively outwards across the slice. As mismatches are always present at each point, it is possible that the full values of contraction stress are developed, corresponding to the maximum thermal strains at each point (accepting that the yield strength $\sigma_{\mathrm{Y}}$ cannot be exceeded anywhere). Note that this interpretation differs from that of Okerblom, in that he attributed the overall longitudinal contraction to integration of compressive plastic strains at each point across the width, due to the plane strain suppression of thermal strain. However, the present formulation in terms of contraction forces gives an identical result.

The overall strain pattern, calculated on the strength of these assumptions, is shown in Fig. 2. The width of the central zone limited by the yield strength is identified by the transverse location where $\alpha T_{\mathrm{m}} \geqslant 2 \varepsilon_{\mathrm{Y}}$. This zone consists of two regions. In the inner region $A$, temperatures exceed the level at which the material has significant yield strength and rapid creep obliterates compressive elastic stress generated during heating. In the outer region $\mathrm{B}$, the material retains strength throughout, but the strain cycle exceeds $2 \varepsilon_{\mathrm{Y}}$. In either case, cooling induces a yield-level tensile residual stress. The outer zone $\mathrm{D}$, which remains elastic throughout the heating and cooling cycle, is defined by $\alpha T_{\mathrm{m}} \leqslant \varepsilon_{\mathrm{Y}}$ and no contraction stress is generated in this region. An indication of the physical positions of these limits in relation to the thermocouple positions in the present test welds is given in Fig. 1 in terms of the non-dimensional parameters. Between these two limits, in zone $\mathrm{C}$, the tensile elastic residual stress is given by $E\left(\alpha T_{\mathrm{m}}-\varepsilon_{\mathrm{Y}}\right)$. Thus the total force generated by contracting material is given by the total shaded area in Fig. 2 multiplied by Young's modulus.

Any convenient method can be used to determine a relationship between $T_{\mathrm{m}}$ and the transverse distance $y$. However the quasi-static analytical treatment of Rosenthal [4] may be used to derive such a relationship in the form

$$
T_{\mathrm{m}}=0.484 \frac{q}{v t} \frac{1}{c \rho} \frac{1}{2 y}\left(1-\frac{\beta y^{2}}{2 \lambda}\right)
$$

If the heat dissipation is small, this reduces to the inversely proportional relationship

$$
T_{\mathrm{m}}=0.242 \frac{q}{v t} \frac{1}{c \rho} \frac{1}{y}
$$




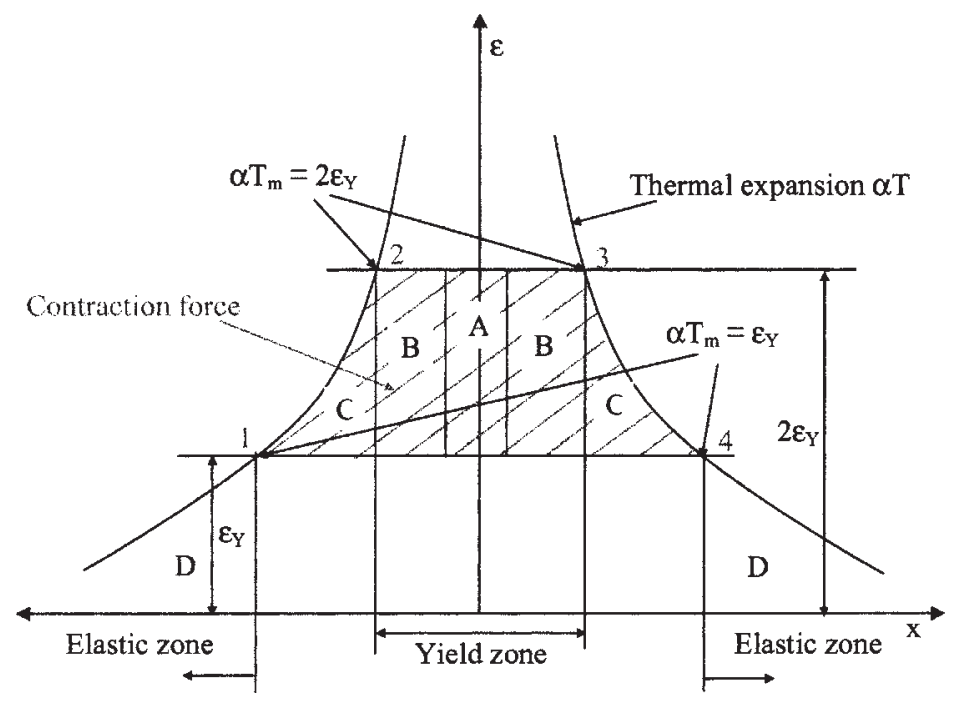

Fig. 2 Formulation of total contraction force via MTS

This relationship was derived by Okerblom [7] using the work of Rykalin and, in reference [8], a correction due to Wells [9] for slower-moving heat sources in a material of high diffusivity was applied to provide a relationship between the transverse distance $y$ and $T_{\mathrm{m}}$ as

$$
T_{\mathrm{m}}=0.242 \frac{q}{v t} \frac{1}{c \rho} \frac{1}{y}-0.4 \frac{\lambda}{v}
$$

The simplest version of these relationships, namely equation (2), can be integrated over the area to provide the force as

$$
F=0.335 \frac{q}{v} \frac{\alpha}{c \rho} E
$$

This result shows several interesting features. Firstly, the critical controlling parameter on longitudinal contraction force is shown to be the heat input rate $(q / v$, in $\mathrm{J} / \mathrm{m})$ of the welding source, as is well known from practice. Secondly, the result is independent of the yield strength. This is a consequence of the fixed yield strength assumption and the inverse relationship between temperature and transverse distance in equation (2). This may not, however, be obtained in practice if the temperature profile differs from the Rosenthal-Rykalin quasi-steady state simplification and the yield strength varies with temperature in the zone of interest. However, this finding suggests, at least, that the dependence of the contraction force on yield strength is weak, which would be an immensely simplifying factor, if shown to be correct.

The assumption of constant thermal and mechanical properties over a typical range of weld cooling seems at first glance to be an oversimplification. However, using properties typical of C-Mn steel, such as a yield strength of $405 \mathrm{MN} / \mathrm{m}^{2}$, Young's modulus of $205 \mathrm{GN} / \mathrm{m}^{2}$ and an expansivity coefficient of $14 \times 10^{-6}{ }^{\circ} \mathrm{C}^{-1}$, the boundary temperatures defining the width of the inner yielded zone and the outer elastic zone are $282^{\circ} \mathrm{C}$ and $141{ }^{\circ} \mathrm{C}$ respectively. If the assumption is correct that distortion is driven mainly by processes occurring in this temperature band, then the variation in properties may not be too significant.

\subsection{Transverse welding deformations}

Okerblom assumed that angular deformation and transverse contractions are due entirely to the thermal contraction of the fused zone, e.g. the triangular zone shown in Fig. 3 of width $b$ and penetration depth $s$ in a plate of thickness $t$.

The implications are that the transverse thermal strains in the remainder of the cross-section are elastic and that there are no restraints on angular deformation, other than that provided by the unwelded area below the fusion zone. The twodimensional treatment here also relies on the finding that the thermal gradient is small along the length of the weld. The simplified treatment arising from this assumption will be designated the 'thermal contraction strain' (TCS) algorithm.

The three diagrams to the right of the weld sketch in Fig. 3 represent the transverse residual stress, the mechanical deformations $\Delta_{\mathrm{m}}$ induced by compression and bending over the width $b$ and the thermal contractions $\Delta_{\mathrm{T}}$ of the fused zone respectively. The starting temperature for the cooling process is $T_{\mathrm{s}}$ and the ratio $k$ defines the proportion of the surface thermal contraction absorbed by constrained plastic flow. The residual transverse deformations arise from a summation of the mechanical and elastic 


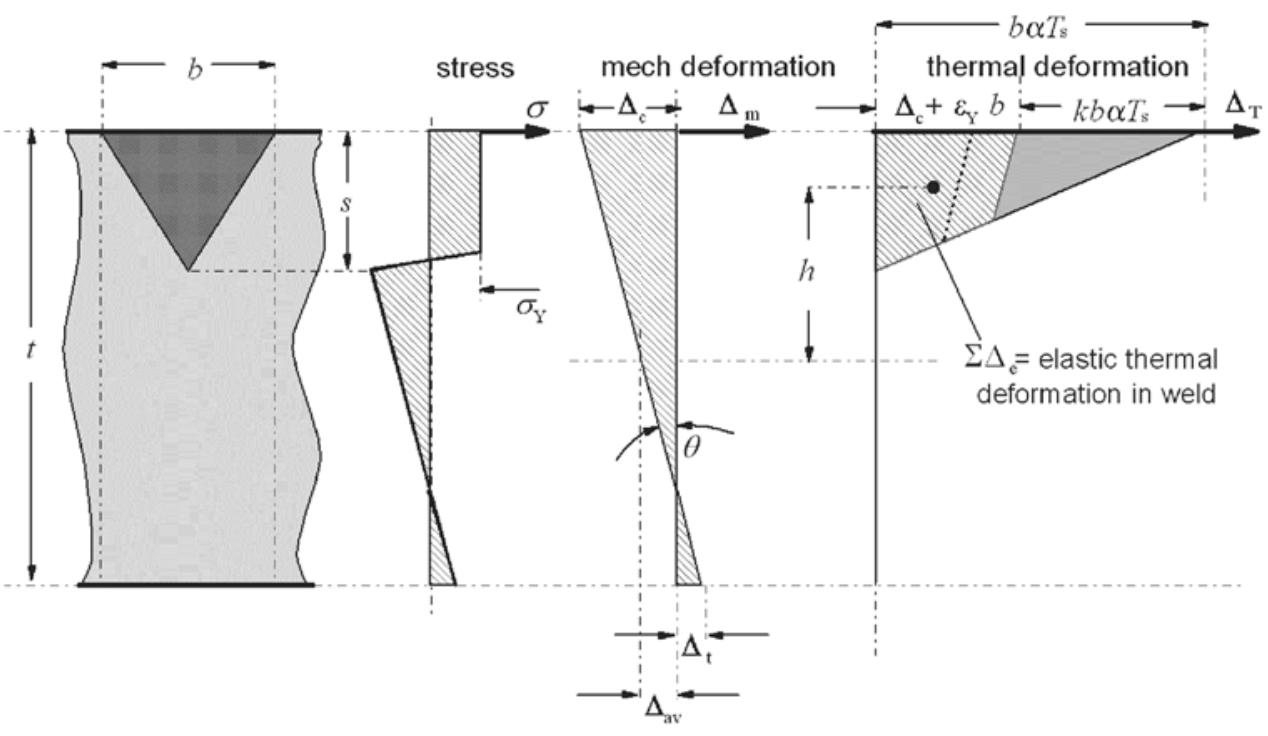

Fig. 3 Schematic diagram of transverse deformation

thermal deformations, leading to the formulations

$$
\begin{aligned}
& \Delta_{\mathrm{av}}=\frac{\sum \Delta_{\mathrm{e}}}{t} \\
& \theta=\frac{\sum \Delta_{\mathrm{e}}}{I} h
\end{aligned}
$$

where $h$ is the distance between the centroids of the mechanical and thermoelastic deformations and $I$ is the second moment of the mechanical deformation area. The total thermoelastic deformation is expressed from the right-hand diagram in terms of the parameter $k$ as

$$
\sum \Delta_{\mathrm{e}}=\frac{1}{2} s b \alpha T_{\mathrm{s}}-\frac{1}{2} s b k^{2} \alpha T_{\mathrm{s}}=\frac{1}{2} s b \alpha T_{\mathrm{s}}\left(1-k^{2}\right)
$$

leading to

$$
\begin{aligned}
& \Delta_{\mathrm{av}}=\frac{1}{2} \frac{s}{t} b \alpha T_{\mathrm{s}}\left(1-k^{2}\right) \\
& \theta=\frac{s}{t} \frac{b}{t} \alpha T_{\mathrm{s}}\left[3\left(1-k^{2}\right)-2 \frac{s}{t}\left(1-k^{3}\right)\right]
\end{aligned}
$$

Noting also that $\Delta_{\mathrm{t}}+\varepsilon_{\mathrm{Y}} b=(1-k) \alpha T_{\mathrm{s}} b$ and $\Delta_{\mathrm{t}}=\Delta_{\mathrm{av}}+\theta t / 2$, these values may be substituted in equations (7) and (8), to solve for $k$ in terms of $s / t$ and $\varepsilon_{\mathrm{Y}} / \alpha T_{\mathrm{s}}$ as shown in Fig. 4. A starting temperature $T_{\mathrm{s}}$ of $100{ }^{\circ} \mathrm{C}$ was chosen for the present application to steel, following the example given by Okerblom, who justified this choice through comparison with tests. However, it may also be observed that this represents an upper bound on the temperature at which steel starts to develop some mechanical strength. In the case of the remaining parameters, the centre curve embodies thermal and mechanical properties appropriate to the present case, as defined in section 2.1.
Note that, when the fusion zone penetrates to approximately 60 per cent of the overall section thickness, the value of $k$ drops to zero and the values from equations (7) and (8) reduce to a case where the fusion zone simply contracts in proportion to its shape from the starting temperature. It is difficult to see that the choice of $1000^{\circ} \mathrm{C}$ starting temperature is realistic for such cases, as thermal contractions at the higher temperatures could be restrained easily by rather small forces, including self-weight and weld joint tacks. This point therefore requires special attention when considering the experimental results.

A similar approach can be applied to other typical fusion zone shapes, such as parabolic and rectangular, as shown in Fig. 5. This confirms practical experience of the effects of weld preparation and fusion zone shape on angular distortion. As angular distortion triggers out-of-plane distortion by displacing the longitudinal contraction force from the neutral axis of the cross-section, it can be understood that fusion zone shape has a profound effect on the overall out-of-plane deformations.

\section{FINITE ELEMENT IMPLEMENTATION}

\subsection{Thermal transients}

The transient temperature profiles in the present study were determined computationally, as outlined in section 1.2, rather than through the analytical treatments described in section 2.1. Conduction has the main effect on the thermal history of the plates during welding, but convection and radiation effects were also modelled, following the scheme described in reference [6]. Non-linear conduction properties were introduced through standard conductivity and enthalpy 


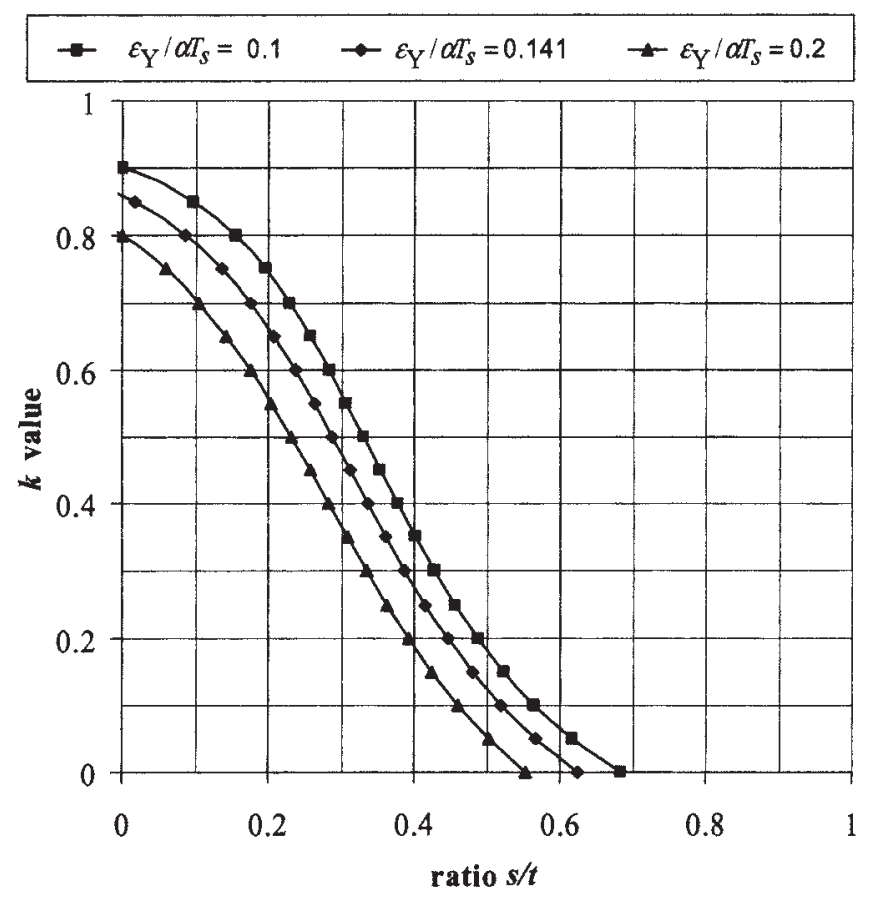

Fig. 4 Relation between $k, s / t$, and $\varepsilon_{\mathrm{Y}} / \alpha T_{\mathrm{s}}$ for a triangular fusion zone. (After reference [7])

data (data obtained in a study on C-Mn plate steel by S. Wen, 2003, personal communication) covering temperatures in the range from ambient to $2000^{\circ} \mathrm{C}$. This approach takes into account phase changes. Alternative computations were carried out using two different configurations of volumetric heat input. In the first, the thermal energy was applied to a fully penetrated V preparation and, in the second, a $0.5 \mathrm{~mm}$ unpenetrated root was simulated. The temperature difference $T_{\mathrm{m}}$ between the two models was no greater than $5^{\circ} \mathrm{C}$ at $20 \mathrm{~mm}$ distance from the weld centre-line.

\subsection{Thermomechanical algorithm}

The MTS and TCS formulations from Okerblom's treatment, as described in section 2, were used to impose longitudinal and transverse strains on elastic finite element models of the large plate assemblies.

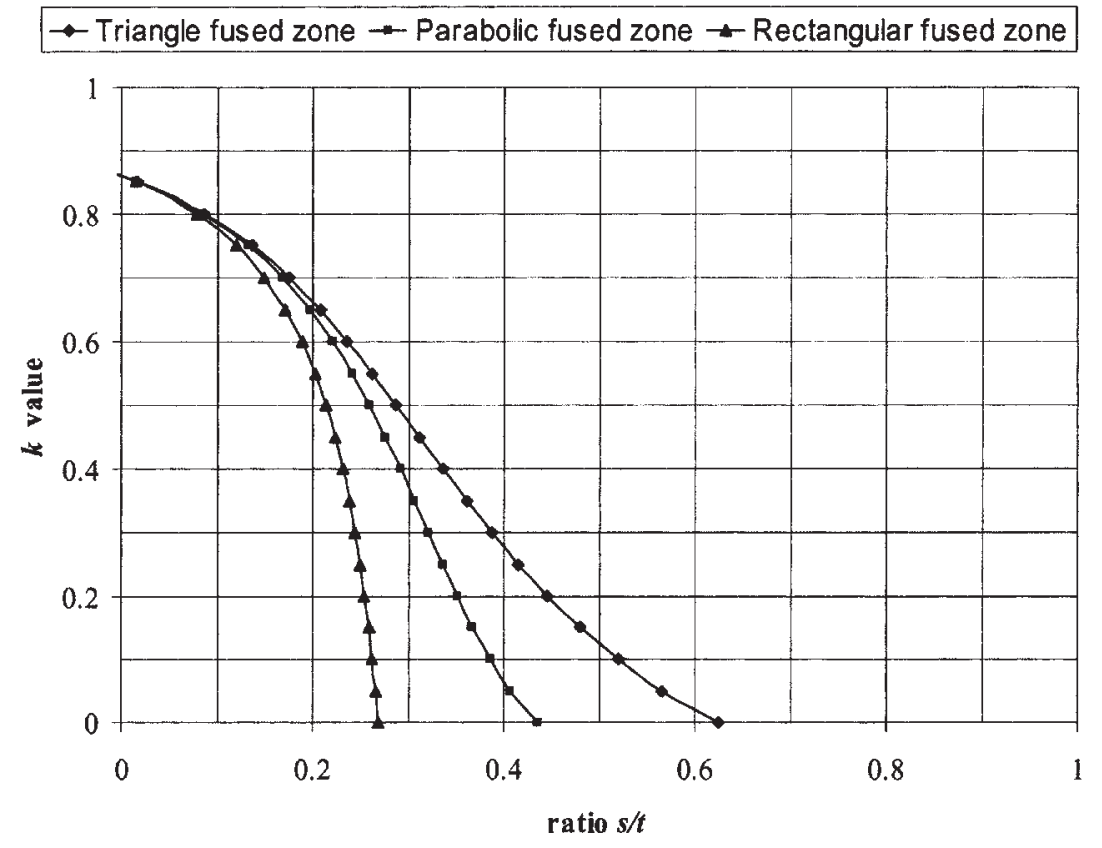

Fig. 5 Relation between $k$ and $s / t$ for various fusion zone shapes. (After reference [7]) 
Introducing an ambient temperature $T_{\mathrm{a}}$, the overall longitudinal contraction in the structural model can be found by applying a 'load' profile corresponding to an artificial temperature reduction equal to $-\left[T_{1}(y)-T_{\mathrm{a}}\right]$ at points $y$ across the width of the plane strain strip. The magnitude of $T_{1}(y)$ is sufficient to develop contraction strain corresponding to $-\varepsilon_{Y}$ in regions A and B of Fig. 2 and zero strain in region D. The limiting node positions $y_{1}$ and $y_{2}$ for these regions are such that $T_{\mathrm{m}}\left(y_{1}\right)=2 \varepsilon_{\mathrm{Y}} / \alpha+T_{\mathrm{a}}$ and $T_{\mathrm{m}}\left(y_{2}\right)=\varepsilon_{\mathrm{Y}} / \alpha+T_{\mathrm{a}}$. In region C, a further simplification was adopted to reduce the element budget and the corresponding computation time. The temperature profile was assumed to follow an inverse relationship with respect to the transverse distance [equation (2)] such that the 'load' temperatures could be defined in terms of a constant $A_{t}$, derived from the computational thermal analysis. Hence, the artificial temperature drop was given by

$$
T_{1}(y)=2 T_{\mathrm{a}}+\frac{\varepsilon_{\mathrm{Y}}}{\alpha}-\frac{A_{t}}{y}
$$

In the case of the transverse contractions, the penetration of the fusion zone profiles in the test welds exceeded the values where the correction parameter $k$ would have been applicable. Hence, the fusion zone was simply defined by the points corresponding to the 'start of cooling' temperature of $1000^{\circ} \mathrm{C}$, these being nearer the centre-line of the weld joint than the points $y_{1}$ defining the width of the yielded zone.

Orthotropic coefficients of expansion were used to enable the artificial temperature loads at a given point to generate the required simulated thermomechanical strains in the finite element implementation. In the longitudinal axis, the actual coefficient of expansion was used but, in the transverse and thickness directions, the coefficient was set to zero, apart from in the fusion zone, where it was set to a value $\alpha_{\mathrm{T}}$ given by

$$
\alpha_{\mathrm{T}}=\left(\frac{T_{\mathrm{a}}-T_{\mathrm{s}}}{T_{\mathrm{a}}-\varepsilon_{\mathrm{Y}} / \alpha}\right) \alpha
$$

The flow chart for the 'simultaneous' algorithm, whereby transverse and longitudinal temperature 'loads' were applied together is given in Fig. 6. The algorithm for the alternative 'sequential' implementation was given in reference [3]. In this case the transverse deformations are applied first and the resulting plate shape, which is distorted angularly along the whole length of the plate, provides the starting point for simulation of the longitudinal deformation process.

\subsection{Other computational features}

The flow chart (Fig. 6) also shows that the computation can be carried out alternatively in terms of a plate assembly which is initially perfectly flat, or one where the initial out-of-plane shape has been measured and is used as the starting point for the simulation. The 'initial shape' sub-algorithm is shown in Fig. 7. This algorithm made use of smoothing procedures detailed in reference [10].

The weight of the plate assembly was supported, as in the real case, at a number of specified points. ANSYS LINK 10 elements, consisting of two-node elements having three translational degrees of freedom per node, were used at the support points. The elements were set to exhibit stiffness under compressive loads but to assume zero stiffness in tension. Non-linear geometry capability was specified, together with the Newton-Raphson line-search sparse-direct equation solver. Figure 8 shows the finite element model, including boundary conditions. Various effects related to choice of Poisson's ratio, coefficient of expansion, fusion zone shape, and load-stepping strategies were investigated to optimize the computational process. The application of the artificial strains within a one-load step analysis significantly reduced the computational time. Typically the simulations required between 2 and $3 \mathrm{~h}$, when performed on a dual-processor workstation with $2.2 \mathrm{GHz}$ central processing unit and 1 Gbyte random-access memory.

Figure 9 shows a sample computed result using the 'simultaneous' algorithm. This assumed that the plate was flat initially but supported at specific points, as described in the next section, so that the weight of the plate generated small out-of-plane deformations. These ripples are magnified by the longitudinal tension in the welding simulation and this is accompanied by an angular distortion of $1.05^{\circ}$.

\section{FULL-SCALE WELDING EXPERIMENTS}

\subsection{Equipment and test assemblies}

The full-scale tests were carried out using a purposebuilt rig (Fig. 10) consisting of several integrated systems with automatic data capture, to carry out the following functions:

(a) to complete a single-pass full-penetration automatic metal-inert gas (MIG) weld with $\mathrm{Ar}-\mathrm{CO}_{2}$ shielding and flux-cored wire, the voltage, current, and travel speed being monitored continuously;

(b) to measure temperatures continuously during welding and cooling, using thermocouples in fixed locations on the plate and a thermographic camera for whole-field recording;

(c) to measure overall out-of-plane transient deformations during welding and cooling, using 


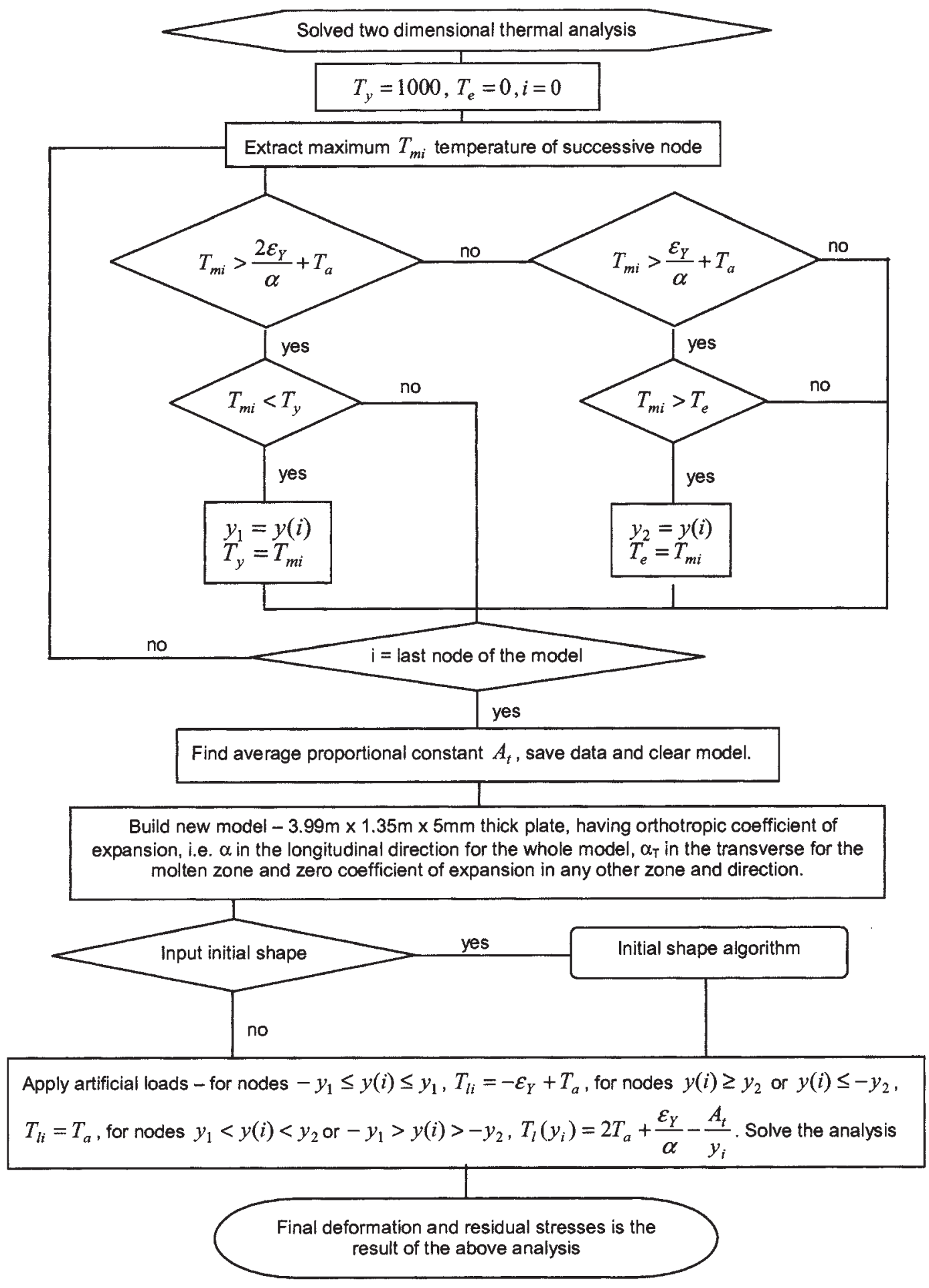

Fig. 6 Thermoelastic 'simultaneous' algorithm

linear variable displacement transducers (LVDTs) in a fixed array (Fig. 11);

(d) to measure out-of-plane deformations over the whole plate, before and after welding and cooling, using a laser scanning system.

The C-Mn steel plates (BS4360 grade 50D) were supported on an array of height-adjustable point supports, fixed to a moving frame in the layout shown in Fig. 11. This frame was traversed at the required welding speed under the welding head and thermographic camera which were mounted in a fixed position. The relative deformation data acquired by the LVDTs were processed to measure the angular distortions and longitudinal profiles over the plate area spanned by the array. 


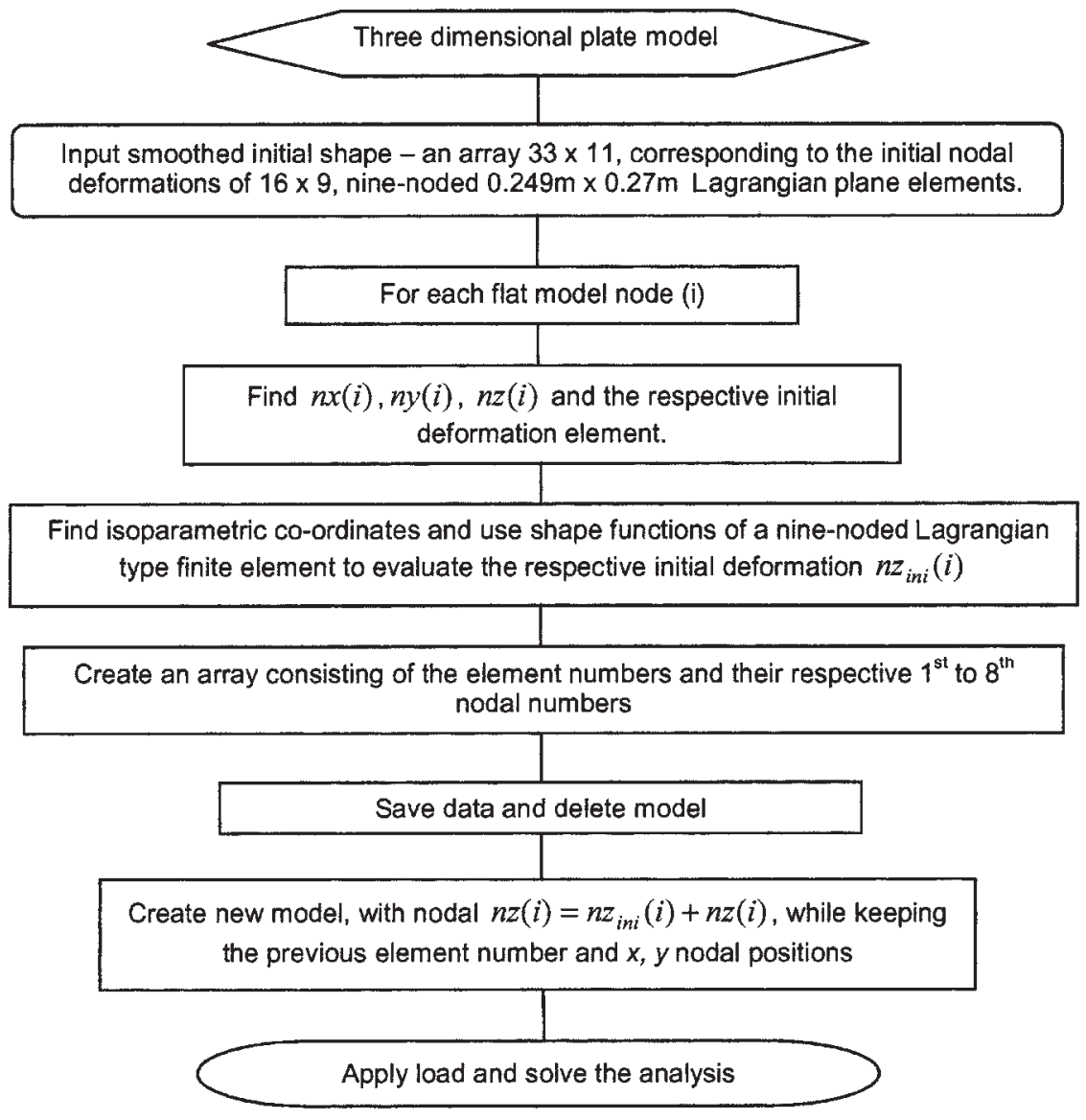

Fig. 7 Initial shape subalgorithm

The MIG weld was applied to a $60^{\circ}$ included angle $\mathrm{V}$ preparation. A ceramic backing strip was used to prevent burn-through, as there was some variation in the root gap. Welding conditions were $26 \mathrm{~V}, 210-225 \mathrm{~A}$, and $5.75 \mathrm{~mm} / \mathrm{s}$ travel speed, giving a nominal gross heat input of $950 \mathrm{~J} / \mathrm{mm}$. Correlation with the thermocouple data indicated a weld energy input efficiency of 70 per cent.

The plate specimens were shot blasted, primed, and laser cut to size by the industrial partner in the project, in order to embody commercially realistic practice in the trials. Typical variations in fit-up and weld tacking procedure were also permitted, to give some indication of sensitivity to such variations. The plates, when offered up for assembly and tacking, proved not to be ideally flat and it was not always possible to align both sides of the joint to a common plane. Likewise, the initial aim to have a tight butt joint with no gap was not achieved throughout and in some cases a gap of 1-2 mm resulted.

Different tack weld assembly strategies were applied, in the light of known variations in industrial procedures and continuously developing evidence from the test welds. In all cases, the run-on-off tabs were fillet welded first, as illustrated in Fig. 10.
Then, in the first three specimens, longitudinal weld tacks were applied consecutively on the underside of the joint at $250 \mathrm{~mm}$ pitch, beginning at the starting edge of the intended weld run. The disadvantage of this procedure was that it proved to be difficult to force the weld preparation edges level, particularly towards the end of the joint. Two measures were applied to deal with this. In specimens 3-6, the tack spacing was increased to $307 \mathrm{~mm}$ pitch (this also reflected advice on typical practice from the industrial partner). Also, for specimens 4 to 6 , a symmetrical tacking strategy was adopted, starting at the centre of the weld run and working outwards, towards both ends. This procedure resulted in an increased tendency to angular distortion in the initial plate shape, but less force was required to bring these specimens into alignment. Eventually, in the two final tests, small mismatches in plate height were simply tolerated, in order to reduce further any 'locked-in' bending moments due to forced levelling and tacking procedures, as applied in specimens 1 to 4 .

The initial out-of-plane deformation patterns of the assembled plates mounted on the rig could fairly be described as unstable, as relatively small out-ofplane forces on the surface of the assembled plates could flip the initial shape from one configuration 


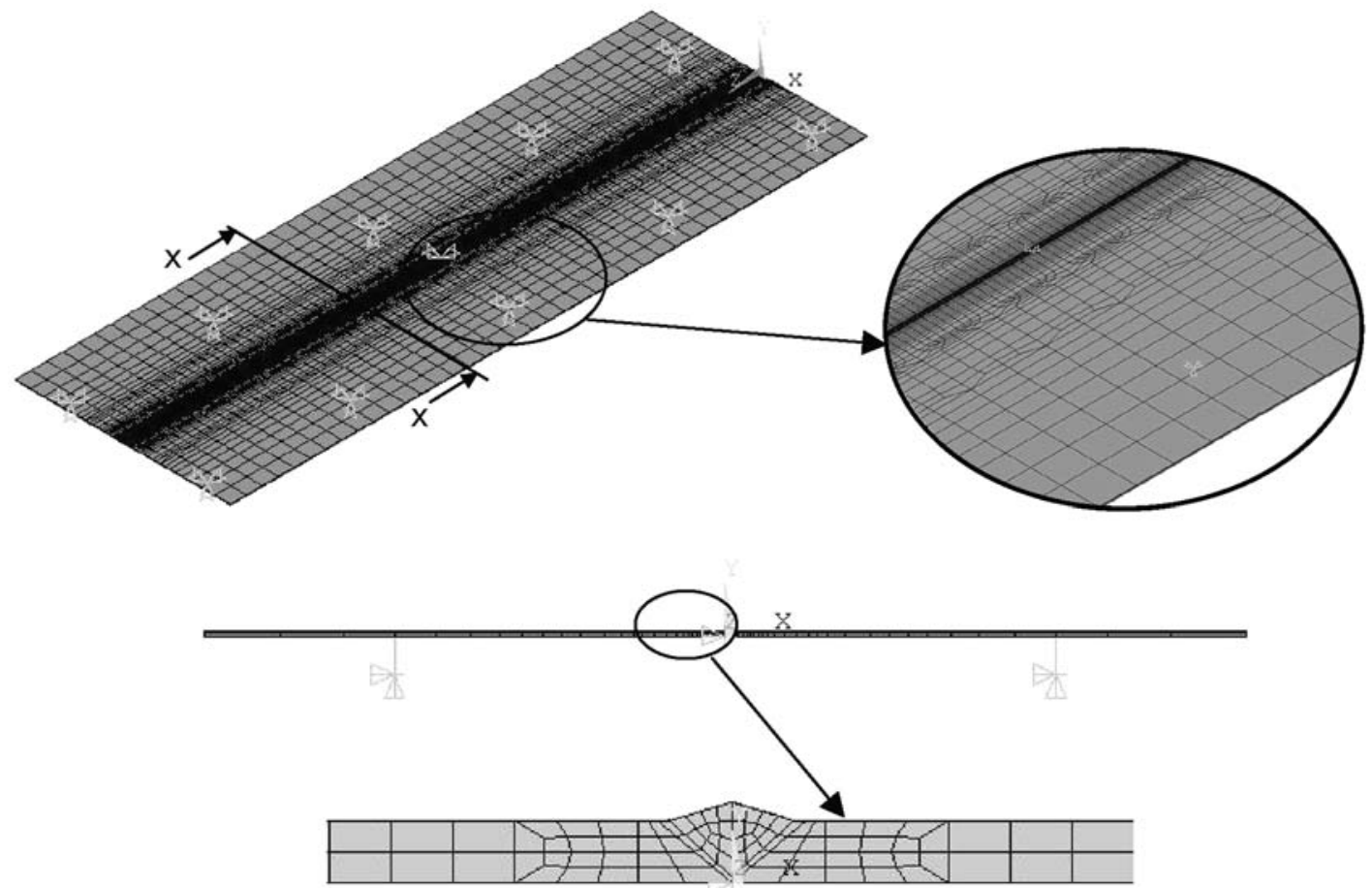

Section X-X

Fig. 8 Finite element modelling of butt welds

to another. This is relevant to the outcomes of each test, as will be noted later.

\subsection{Results and discussion}

The measured deformation behaviour of the large butt-welded plates was found to be complex.
Variations between different test specimens were identified, due to initial shape imperfections, effects related to initial assembly and weld tacking procedures, transient mechanical variations in boundary support conditions, and variations in the welding conditions. These variations led to inconsistencies in measured distortions between the different tests.

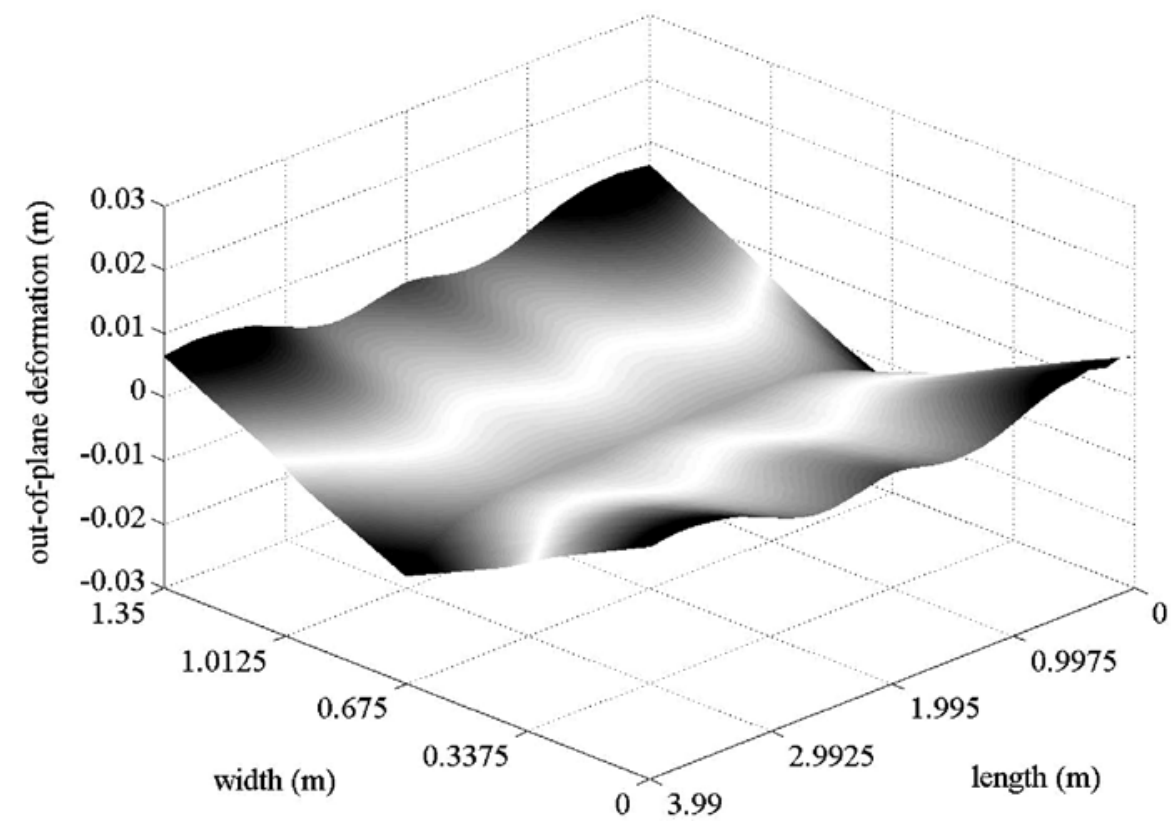

Fig. 9 Computed out-of-plane deformation of an initially flat plate 


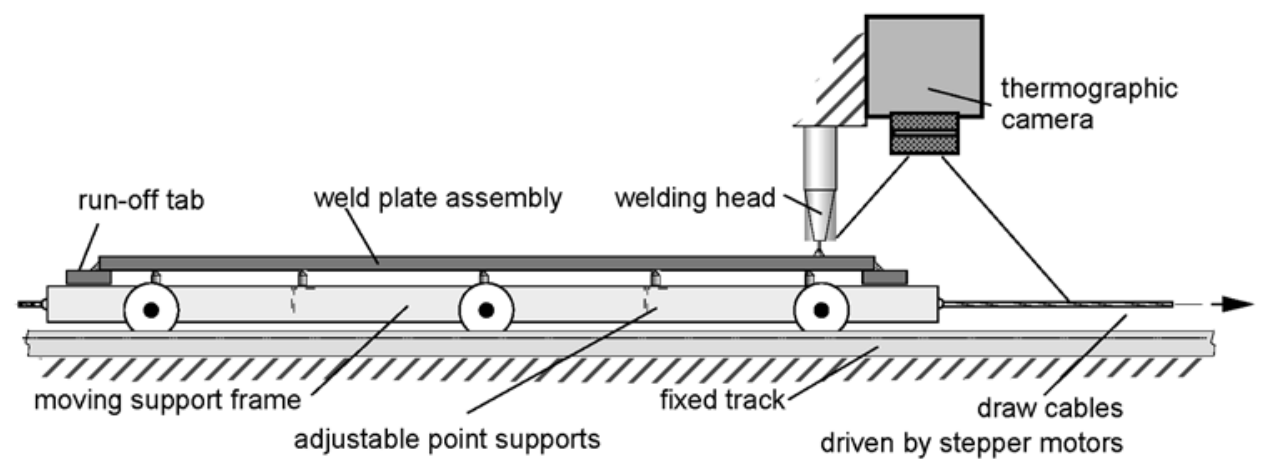

Fig. 10 Schematic diagram of welding rig

These effects are nevertheless encountered normally in industrial practice and therefore represent a realistic challenge for the simulation of welding. The experimental programme highlighted the fact that most of the final out-of-plane movement takes place long after the weld has been completed. This finding implies that detailed knowledge of high-temperature material properties may not be crucial to the simulation process (as it would be for welding process simulation of metallurgy-dependent phenomena).

The net out-of-plane deformations (final minus initial deformations) varied substantially for each specimen in the series. Test specimens 2 and 4 were particularly eccentric, as the final $0.5 \mathrm{~m}$ of these plate assemblies flipped from positive to negative angular deformation, leaving a severe transverse fold at the transition point (as shown in Fig. 12). It should be noted that, in this figure, the zero coordinate on the 'length' axis corresponds to the start-of-weld position. The reasons for this behaviour will be discussed later.

Aside from these two cases, the deformation patterns were characterized largely as magnifications of the initial deformations and these were simulated reasonably by the computational model. Figure 13 shows a direct comparison between the test result for specimen 6 and the corresponding 'simultaneous' computation. This is one of the most accurate results and, although the computation underestimates the deformations a little, it replicates the complex pattern well.

The capability of the computational method to model angular deformation is particularly important, as the resulting cross-sectional shape drives the longitudinal behaviour. Figure 14 shows overall contour maps of angular deformation in specimen 5 and again indicates good comparison. The overall root-meansquare (r.m.s.) values of experimental and calculated angular deformations are almost identical, although such a measure allows differences at each point, as observed. It is also noteworthy that the angular deformations in the experimental case are highly concentrated in the fusion zone, as assumed in the simple Okerblom model for transverse deformation.

However, there were discrepancies between the experimental and calculated deformation patterns, not least for specimens 2 and 4, and it is of interest to determine the reasons. Figure 15 shows the development of angular deformation and curvature

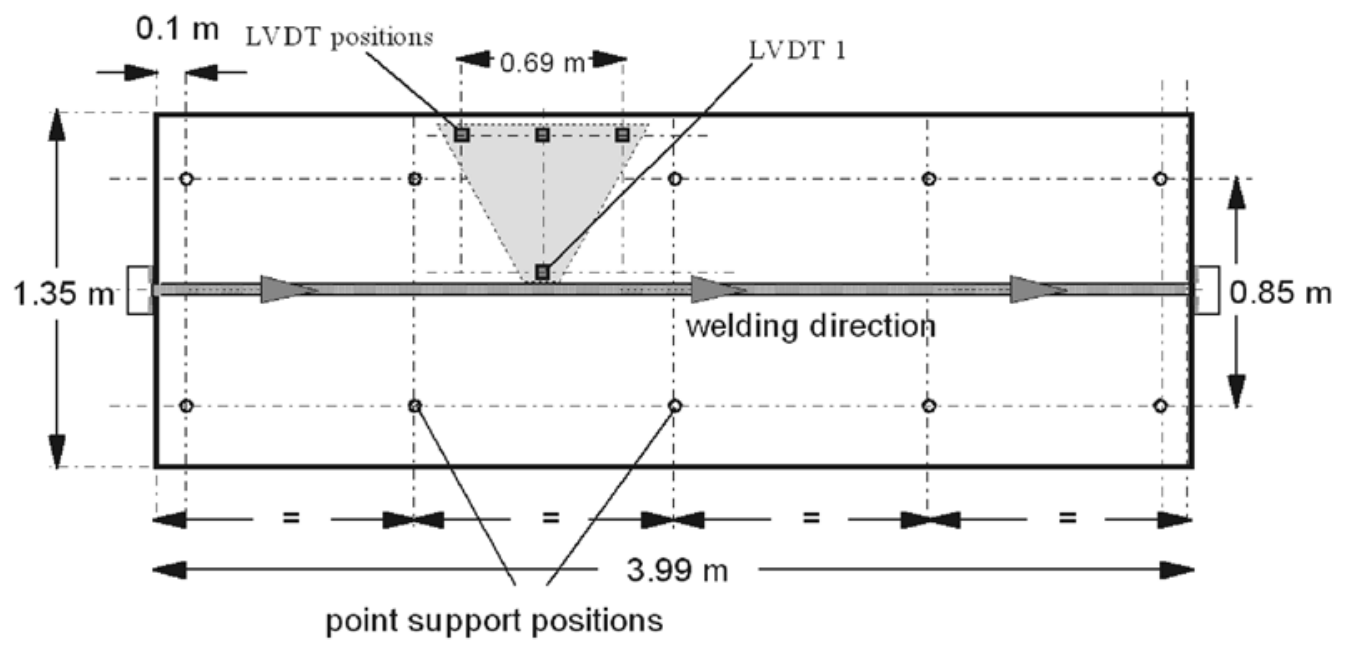

Fig. 11 Layout of supports and LVDTs 


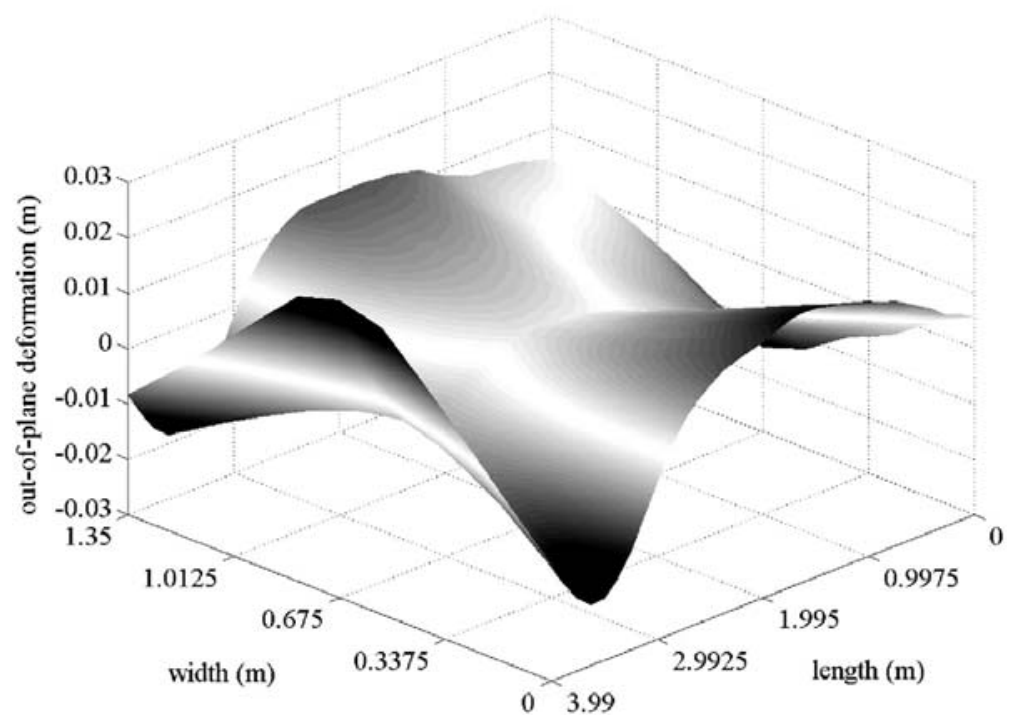

Fig. 12 Net out-of-plane deformation: specimen 4
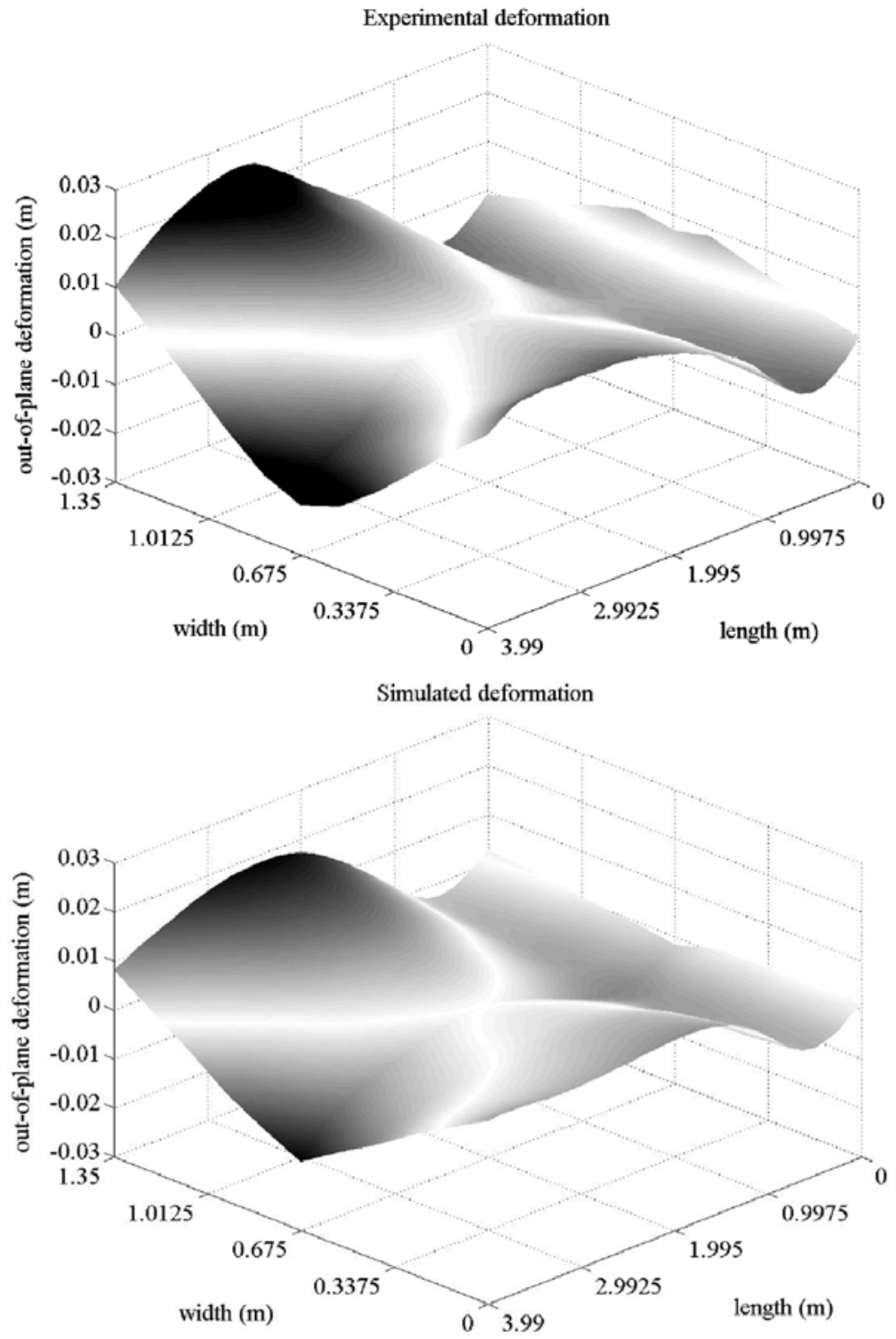

Fig. 13 Experimental and calculated deformations: specimen 6 

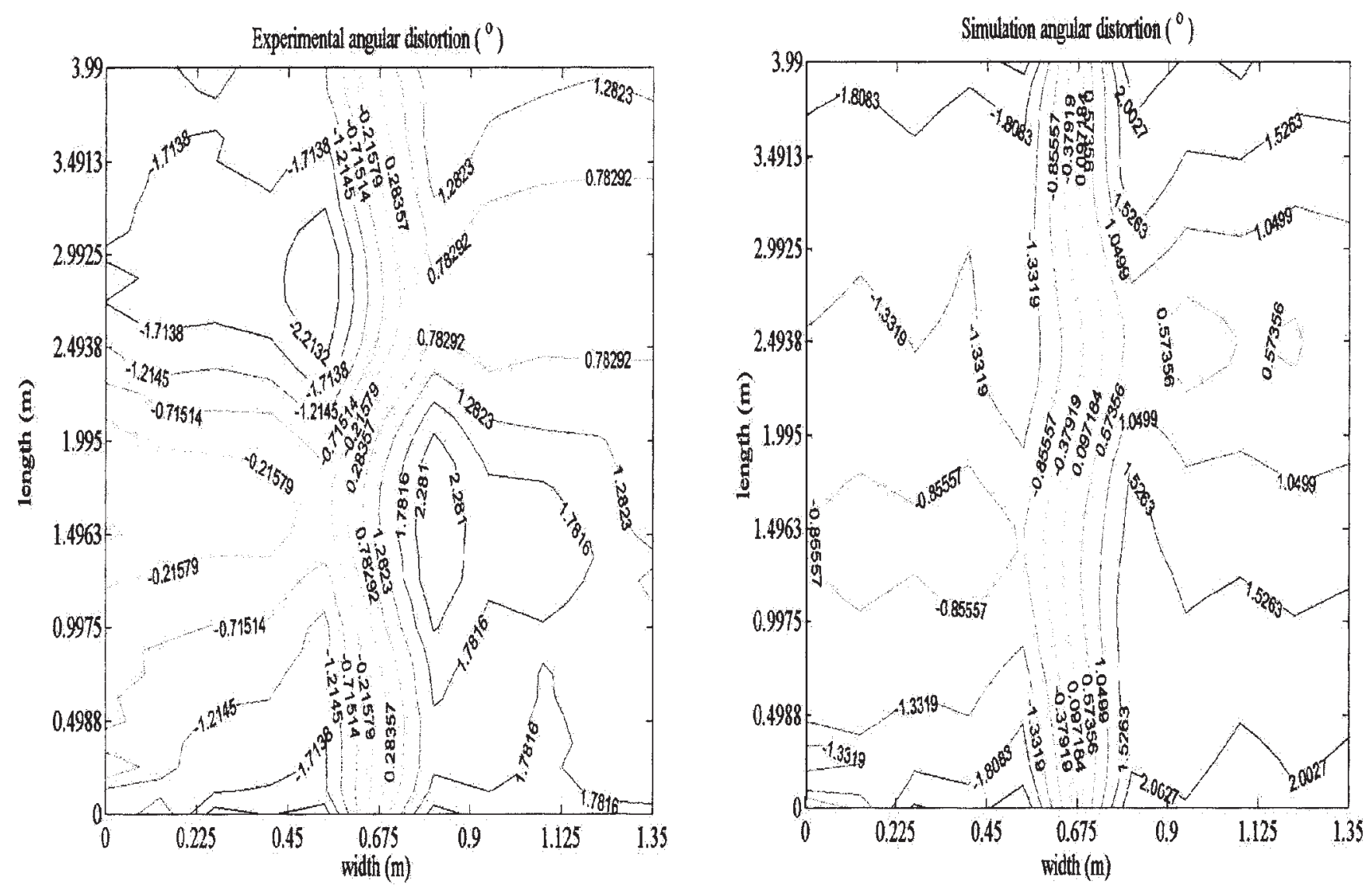

Fig. 14 Experimental and calculated angular deformations: specimen 5

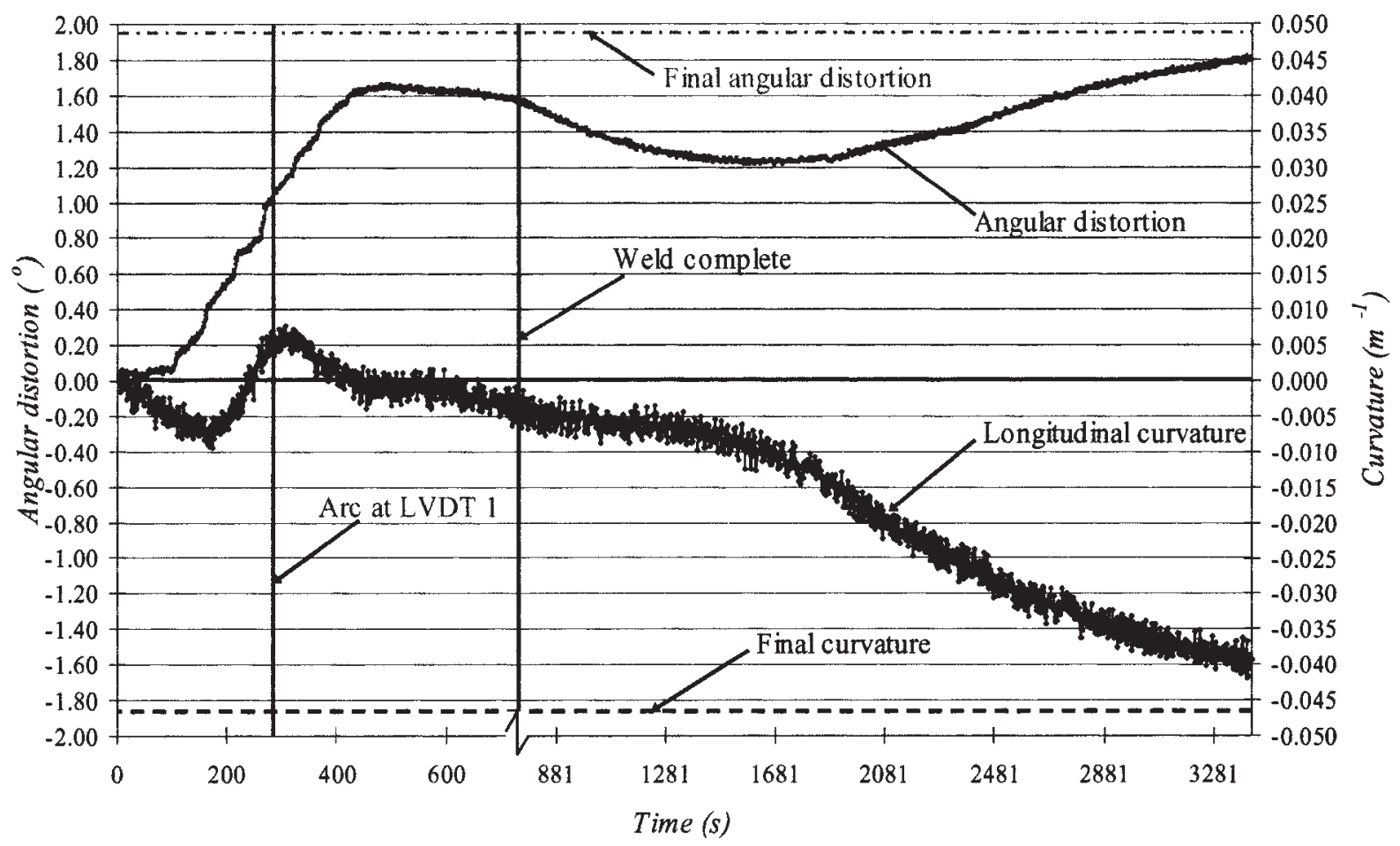

Fig. 15 Dynamic distortion results: specimen 5 


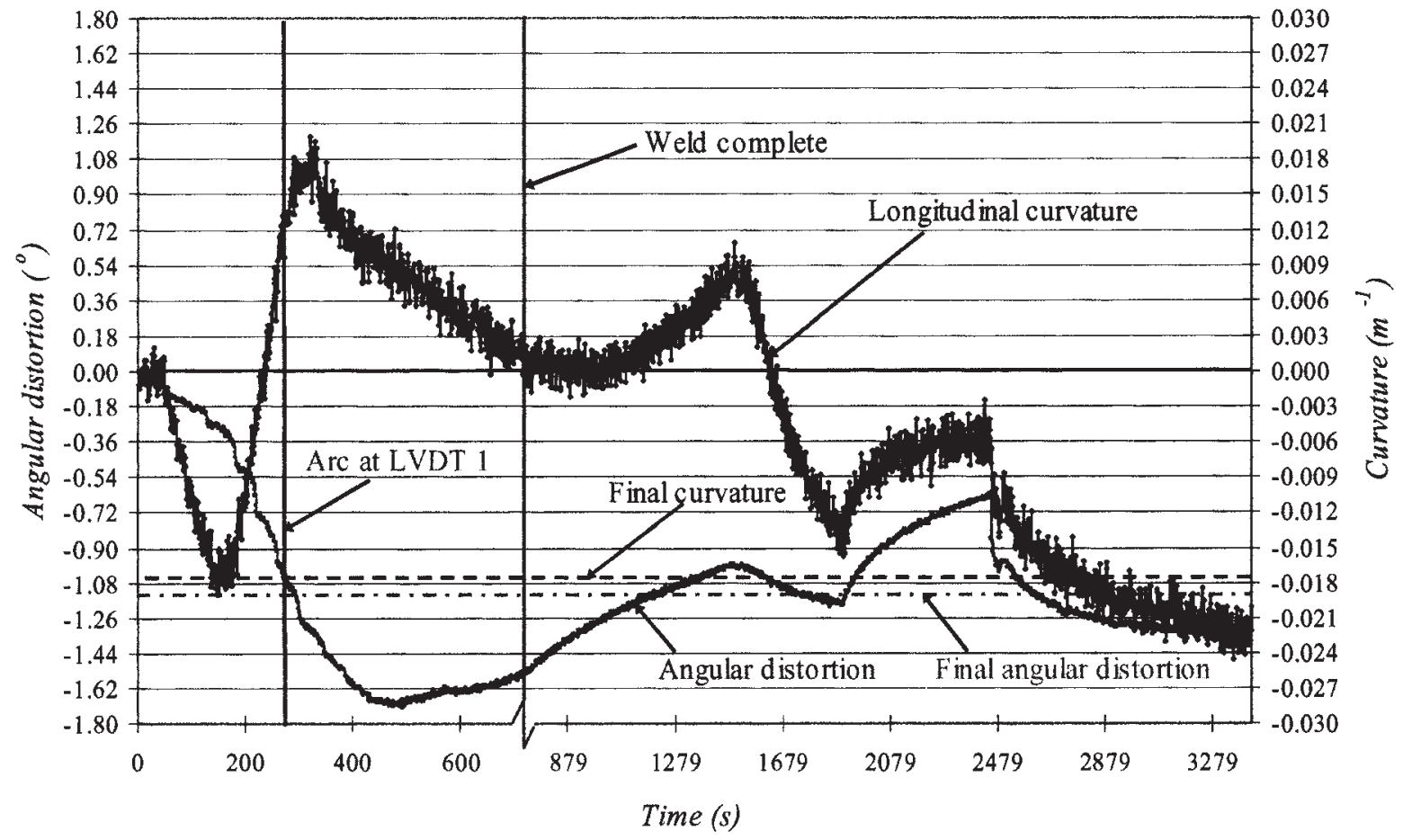

Fig. 16 Dynamic distortion results: specimen 2

during test 5 , in the region covered by the measurement system (see Fig. 11). As the heat source passes, the assembly deforms quickly into a V-shape and continues to do so more slowly until the whole plate has cooled to ambient temperature over a long period. The step changes in angular deformation, which can be seen in the trace, are found in all the tests and correspond exactly to the points where tack welds were fused, as the heat source passed. This event releases the plates to the influence of the transverse contraction forces in the trailing region of the weld. In the case of longitudinal curvatures (negative curvature implies 'hogging' of the plate) there are minor variations during welding, but most of the eventual curvature in this region takes place during the long period of cooling to ambient temperature. The changes in curvature rate and direction during welding coincide with the points where the heat source crosses the transverse axis of each support pair, implying that the plate is lifting off various supports, as indeed was observed. Also note that sagging curvature is promoted when the heat source passes through the monitored region. This is expected, as a consequence of the $\mathrm{V}$-shape and the relative expansion of the weld zone during this interval.

The pattern of dynamic deformation behaviour in test 2, which was unstable, is shown in Fig. 16. The curvature changes are much more exaggerated but also relate to the support positions. In this case, the implication is that changes in curvature after weld completion have been enough to raise the plate off the final pair of supports and the cantilevered weight of overhanging plate has triggered an unstable reversal of angular deformation and curvature step at the 2479 s marker.

The variation in tacking procedure is probably also an important factor leading to divergent behaviour in the tests, as the action of aligning the plate edges will generate initial transverse moments which are both variable and impracticable to incorporate in the model. It is probably significant that the two results that were best simulated were from the tests where no clamping was applied.

In the case of these two abnormal test outcomes, improved simulation might be achieved by stepping the artificial thermal load, thereby allowing the variation in support conditions to influence the buckling modes of the plate assemblies. Similar measures would be necessary to take account of in-built edge moments due to initial misfitting of the butt edges, which were forced into alignment and tacked.

Overall comparison of calculated and test results is difficult to characterize in the light of these variations but Table 1 provides a measure of comparison through r.m.s. values. The values in the error columns represent the r.m.s. differences between experimental and calculated values at discrete points on the measurement grid and the remaining data represent the r.m.s. values of all deformations in the plates. (In the case of specimens 2 and 4, the data from the end sections of the plates, which flipped to the opposite angular deformation state were missed out from the r.m.s. determination.) 
Table 1 Comparison of test results and calculations (r.m.s. basis)

\begin{tabular}{|c|c|c|c|c|c|c|}
\hline Specimen & $\begin{array}{l}\text { Experimental } \\
\text { out-of-plane } \\
\text { deformation } \\
(\mathrm{mm})\end{array}$ & $\begin{array}{l}\text { Simulation } \\
\text { out-of-plane } \\
\text { deformation } \\
(\mathrm{mm})\end{array}$ & $\begin{array}{l}\text { Error } \\
(\mathrm{mm})\end{array}$ & $\begin{array}{l}\text { Experimental } \\
\text { angular } \\
\text { deformation } \\
\text { (deg) }\end{array}$ & $\begin{array}{l}\text { Simulation } \\
\text { angular } \\
\text { deformation } \\
\text { (deg) }\end{array}$ & $\begin{array}{l}\text { Error } \\
\text { (deg) }\end{array}$ \\
\hline 1 & 7.16 & 5.18 & 5.88 & 1.23 & 1.30 & 0.82 \\
\hline 2 & $\mathrm{n} / \mathrm{a}^{*}$ & 5.11 & $\mathrm{n} / \mathrm{a}^{*}$ & $\mathrm{n} / \mathrm{a}$ & 1.28 & $\mathrm{n} / \mathrm{a}$ \\
\hline 3 & 8.20 & 5.14 & 7.59 & 1.42 & 1.33 & 0.92 \\
\hline 4 & $\mathrm{n} / \mathrm{a}^{*}$ & 6.00 & $\mathrm{n} / \mathrm{a}^{*}$ & $\mathrm{n} / \mathrm{a}$ & 1.46 & $\mathrm{n} / \mathrm{a}$ \\
\hline 5 & 7.11 & 5.55 & 3.9 & 1.38 & 1.39 & 0.49 \\
\hline 6 & 8.83 & 6.55 & 3.20 & 1.45 & 1.18 & 0.57 \\
\hline
\end{tabular}

n/a, not available.

*See text.

\section{CONCLUSIONS AND SUMMARY}

The main aim of this study was to investigate a simplified finite element simulation of out-of-plane distortion, caused by the process of fusion butt welding. The key simplifications in this approach are embodied in analytical treatments of the angular and longitudinal deformations through the TCS and MTS algorithms respectively.

From the computational viewpoint, the problem has been reduced to an elastic finite element structural model, with an artificial temperature loading, applied in a single load step. This was relatively simple to implement and solved quickly. The 'sequential' and 'simultaneous' alternative formulations of the interacting angular and longitudinal deformations gave slightly different results, the latter probably being the more realistic.

Comparison between the results of the simulations and the out-of-plane deformations measured was good, bearing in mind the substantial variations found in practice, as discussed in section 4.2. Overall shape comparisons were good, except for two specimens where other factors intervened. The magnitudes of angular deformations and overall out-of-plane deformations were predicted well enough to be useful as a guide to what would happen in a practical case. If anything, the simulations slightly underpredicted the real deformations.

The principal value of the simulation process therefore is that it provides a background prediction, which gives insight into the causes of distortion and a tool to formulate possible remedies. For example, it is clear that initial shape imperfections are crucial to the process of prediction, as also are in-built edge moments and support conditions, especially for flexible plates subject to gravitational out-of-plane forces.

The thermal simulation aspect of the programme has not been discussed in detail, but it is worth noting that a relatively simple two-dimensional treatment was effective in terms of describing the thermal transients, at least to the extent necessary to provide input values to the thermoelastic simulation. The prediction accuracy does depend, however, on some means of correlating the overall simulated heat input to the actual weld power.

Summarizing, the following conclusions can be drawn:

1. A finite element computational procedure for simulating the process of industrial-scale fusion butt welding has been demonstrated, whereby the thermoelastic-plastic part of the computation is replaced by simple analytical algorithms. Nonlinear structural behaviour arises in this type of thin flat geometry and a non-linear displacement analysis capability is essential.

2. The thermal part of the simulation scheme was based on simplified two-dimensional finite element modelling. The thermocouple and thermographic measurements of temperature confirmed that this approach was adequate for the test cases, which involved high-power fast-moving heat sources.

3. The simulation results for out-of-plane deformations compared well with full-scale welding trials, in terms of both overall shape characterization and magnitudes of angular and overall deformations.

4. The success of the simulation process clearly depends on the quality of input data and it was found, not surprisingly, that input information on the initial shape of the test pieces was crucial to accurate prediction. It was also clear that deformations due to self-weight were important, both in the initial state and transiently during the welding and cooling process. An advantage of the simplification inherent in the procedure is that the available computing power can be directed to modelling of the structural features which have a powerful influence on the deformation behaviour.

\section{ACKNOWLEDGEMENTS}

The project has been supported by the Engineering and Physical Sciences Research Council and BAE 
Systems Marine. The authors gratefully acknowledge the participation in the project of $\mathrm{Dr}$ Norman MacPherson of BAE Systems and colleagues at the University of Strathclyde, Alex Galloway, Dr Chee Kong Lee, and Henry Tan.

\section{REFERENCES}

1 Huang, T. D., Dong, P., DeCan, L. A., and Harwig, D. D. Residual stresses and distortions in lightweight ship panel structures, Northrop Grumman. Technol. Rev. J., 2003, 11(1), 1-26.

2 Bruce, G. J., Yuliadi, M. Z., and Shahab, A. S. Towards a practical means of predicting weld distortion. SNAME J. Ship Prod., May 2001, 17(2), 62-68.

3 Camilleri, D., Comlekci, T., and Gray, T. G. F. Out-ofplane distortion of CMn steel plates during flux-cored $\mathrm{CO}_{2} / \mathrm{Ar}$ automatic butt welding. In Proceedings of the International Conference on Metal Fabrication and Welding Technology (METFAB-2003), September 2003, pp. 117-127.

4 Rosenthal, D. The theory of moving sources of heat and its application to metal treatments. Trans. Am. Soc. Mech. Engrs, 1946, 68(8), 849-866.

5 Lindgren, L. E. Finite element modeling and simulation of welding. Part 1: increased complexity. J. Thermal Stresses, 2001, 24, 141-192.

6 Camilleri, D., Comlekci, T., Lee, C. K., Tan, H., and Gray, T. G. F. Investigation of temperature transients during flux-cored $\mathrm{CO}_{2} / \mathrm{Ar}$ butt welding of $\mathrm{CMn}$ steel plates. In Proceedings of the International Conference on Metal Fabrication and Welding Technology (METFAB-2003), September 2003, pp. 107-116.

7 Okerblom, N. O. The Calculations of Deformations of Welded Metal Structures, 1958 (Her Majesty's Stationery Office, London).

8 Wickramasinghe, D. M. G., and Gray, T. G. F. A simple treatment of welding distortion. Weld. Res. Int., 1978, 8(5), 409-422.

9 Wells, A. A. Heat flow in welding. Weld. J., Weld. Res. Suppl., 1952, 31, 263s-267s.

10 Comlekci, T., Camilleri, D., and Gray T. G. F. Finite element representation of experimental surface deformation data from fusion welded plates. In Proceedings of the 11th Annual Conference of the Association for Computational Mechanics in Engineering, Glasgow, 2003, pp. 33-36.

\section{APPENDIX}

\section{Notation}

$A_{t} \quad$ temperature distribution coefficient [see equation (9)]

$b \quad$ fusion zone width (see Fig. 3)

$c$ specific heat

E Young's modulus

$h \quad$ centroid offset (see Fig. 3)

I transverse second moment of area (see Fig. 3)

$k \quad$ angular contraction parameter (see Fig. 3)

$s \quad$ penetration depth (see Fig. 3)

$t \quad$ plate thickness

$T_{\mathrm{a}} \quad$ ambient temperature

$T_{1} \quad$ cooling temperature for a longitudinal artificial load

$T_{\mathrm{m}} \quad$ maximum temperature at the $y$ coordinate from thermal analysis

$T_{\mathrm{s}} \quad$ starting temperature for the angular deformation process

$v \quad$ weld travel speed

$x, y \quad$ longitudinal and transverse coordinates respectively in the plate

$\alpha \quad$ expansivity

$\alpha_{\mathrm{T}} \quad$ artificial expansivity for transverse deformations

$\beta \quad$ coefficient of loss of temperature

$\Delta_{\mathrm{av}}$ average transverse mechanical deformation (see Fig. 3)

$\Delta_{\mathrm{c}} \quad$ transverse mechanical deformation (compressive) (see Fig. 3)

$\Delta_{\mathrm{e}} \quad$ transverse elastic deformation in the weld (see Fig. 3)

$\Delta_{\mathrm{m}} \quad$ transverse mechanical deformation (see Fig. 3)

$\Delta_{\mathrm{t}} \quad$ transverse mechanical deformation (tensile) (see Fig. 3)

$\Delta_{\mathrm{T}} \quad$ thermal deformation (see Fig. 3)

$\varepsilon_{\mathrm{Y}} \quad$ yield strain

$\theta \quad$ angular deformation (see Fig. 3)

$\lambda$ thermal diffusivity

$\rho$ density

$\sigma_{\mathrm{Y}} \quad$ yield strength 